\title{
METASTASIS-ASSOCIATED C4.4A, A GPI-ANCHORED PROTEIN CLEAVED BY ADAM10 AND ADAM17
}

\author{
C.W. Esselens ${ }^{1}$, J. Malapeira ${ }^{1}$, N. Colomé ${ }^{1,2}$, W. Jabs ${ }^{3}$, D. Suckau ${ }^{3}$, \\ C. Baessmann ${ }^{3}$, J. Arribas ${ }^{1}, \underline{\text { F. Canals }}^{1,2}$ \\ Medical Oncology Research Program ${ }^{1}$ and Proteomics Laboratory ${ }^{2}$, \\ Vall d'Hebron University Hospital Research Institute, Barcelona, Spain \\ Bruker Daltonik GmbH, Bremen Germany
}

Metalloproteases play a complex role in tumor progression. Proteomic approaches to identify the array of substrates of a given metalloprotease (degradome), can help to unveil its role in tumor growth and metastasis. Here we describe a proteomic screening to compare the proteins secreted by MCF7 cells, derived from an invasive mammary tumor, and the same cells expressing shRNAs that knock down ADAM10 or -17. Cells grown in conditions were the protease is expressed or knocked down were differentially labeled, by incorporating isotopically labeled amino acids (SILAC). Glycoproteins from the conditioned media of each of the two cell cultures were purified by affinity chromatography. The samples were then pooled and run on a 1D SDS-PAGE gel. The gel lane was then cut into 20 fractions and digested. Each fraction was analyzed by RP-LCMS/MS. Protein identification and quantification of relative abundances was performed using WARP-LC, an integrated software platform for LC-MS/MS workflows.

A number of known substrates of both proteases were identified as such in the analysis, showing the expected decrease of the shed extracellular domain abundance in the medium upon knock down of the protease. Thus, the cell-adhesion proteins E-cadherin and Desmoglein-2, or the PTP receptor-type k, were identified as substrates of ADAM10. Fractalkine and NCAML1 were found as substrates of ADAM17.

In addition several new candidate substrates of both proteases were identified. Among them, the GPI-anchored protein C4.4A, was identified and further validated as substrate of both ADAM10 and ADAM17 proteases. According to the identified peptides, both proteases cleave this protein close to the juxtamembrane region, releasing a soluble form devoid of the GPI-anchor. C4.4A protein, homologous to the urokinase-type plasminogen activator receptor, has been related to tumor invasion and metastasis. Cleavage of this protein by ADAMs constitutes a previously unknown level of regulation of its function. 